\title{
MOdèles EXPÉRIMENTAUX DE TOXOPLASMOSE. Applications pharmacologiques
}

\author{
DEROUIN F.*, LACROIX C.*, SUMYUEN M.H.*, ROMAND S.** \& GARIN Y.J.F.*
}

Summary : EXPERIMENTAL MODELS OF TOXOPLASMOSIS. PHARMACOLOGICAL APPLICATIONS

Toxoplasma gondii is an ubiquitous protozoan parasite causing severe or life-threatening infections in immunocompromised patients and in congenitally infected infants. Animal models have been extensively used to describe the pathology of infection and to identify new effective drugs for the treatment of congenital infec tions, chrorioretinitis and toxoplasmic encephalitis. Although inherent differences between man and animal can reduce the relevance of data obtained experimentally, animal models have greatly improved our knowledge on the various aspects of toxoplasmosis. Toxoplasma infection can be easily obtained in most laboratory animals, with exception of rats which are partially resistant. According to the strain used, the resulting infection may be acute, subacute or chronic, and can be monitored either by the survival of animals, the histopathological examination of lesions or, preferably, by titration of parasites in infected tissues using subinoculation to mice or tissue culture. This latter method has proved particularly useful to describe the kinetics of infection in host tissues and to assess the efficacy of drugs, according to their pharmacokinetics and tissue distribution.

The relevance of results obtained in animal models of congenital toxoplasmosis and of chrorioretinitis is more questionable, due to the marked differences between the mode of infection in humans and in animals. Experiments performed in primates provided valuable informations for the management of therapy of congenital toxoplasmosis but were of limited interest for ocular toxoplasmosis. The pathogeny of toxoplasmic encephalitis is still poorly understood, and no experimental model is fully satisfactory to produce focal encephalitic lesions as observed in immunocompromised humans. Acute infections with highly virulent strains induce disseminated infection with major pulmonary and brain involvement, and thus can be used to assess the efficacity of drugs in these tissues. Direct inoculation of tachyzoites into brain tissue can induce focal encephalitis but this model is of difficult use for large scale studies Although cellular immunity is mainly responsible for the control of toxoplasmosis at the chronic stage, administration of immunosuppressive drugs does not usually result in focal brain reactivation; such reactivation can only be obtained using antibodies against CD8 and CD4 T lymphocytes or interferon gamma. Another experimental approach is the use of genetically immunodeficient animals : these models are of limited interest for pharmacological research since infection of nude or $T$ depleted mice usually results

\section{Résumé}

Toxoplasma gondii est un protozoaire parasite ubiquiste, responsable d'infections sévères, voire mortelles chez les sujets immunodéprimés et en cas de contamination congénitale. Les modèles expérimentaux animaux ont été très largement utilisés pour étudier le pouvoir pathogène de ce parasite et rechercher de nouveaux médicaments pouvant être utilisés pour le traitement des infections congénitales, des choriorétinites et des encéphalites toxoplasmiques. Bien que des différences évidentes entre l'homme et les animaux limitent l'intérêt des études réalisées chez l'animal, les modèles expérimentaux ont largement contribué à une meilleure connaissance de la pathogénie de la toxoplasmose. Toxoplasma gondii peut infecter facilement la plupart des animaux de laboratoire, à l'exception du rat qui est partiellement résistant. En fonction de la souche utilisée, il est possible d'obtenir une infection aiguë, subaiguë ou chronique dont le suivi peut être réalisé par l'étude de la survie, l'examen histopathologique des lésions ou, de préférence, par le titrage parasitaire dans les tissus, par subinoculation à l'animal ou par culture cellulaire. Cette dernière méthode s'est avérée particulièrement utile pour décrire la cinétique de l'infection chez l'hôte et démontrer le mode d'action des médicaments anti-parasitaires en fonction de leur pharmacocinétique et de leur distribution tissulaire.

La possibilité d'extrapoler à l'homme les résultats obtenus dans les modèles expérimentaux de toxoplasmose congénitale ou de toxoplasmose oculaire reste limitée par le fait que les modes de contamination expérimentale différent sensiblement chez l'animal. Seules les études réalisées chez les primates sont très informatives sur la thérapeutique de la toxoplasmose congénitale, mais elles sont d'un faible intérêt dans la toxoplasmose oculaire.

La pathogénie de la toxoplasmose cérébrale reste encore mal connue, et aucun modèle expérimental ne permet de réaliser chez l'animal les lésions tissulaires focalisées observées chez l'homme. L'infection aiguë par une souche de haute virulence entraîne une dissémination parasitaire, avec une atteinte à prédominance pulmonaire et cérébrale; ce type de modèle peut être utilisé pour évaluer l'efficacité des médicaments dans ces foyers infectieux. L'inoculation directe de tachyzoites dans le tissu cérébral permet d'obtenir des foyers parasitaires focalisés, mais ce modèle n'est pas applicable aux études pharmacologiques utilisant un grand nombre d'animaux. Bien que l'immunité cellulaire soit responsable du contrôle de l'infection, surtout à la phase chronique, les traitements par des médicaments immunosuppresseurs n'entraînent pas de réactivation; celle-ci peut être obtenue par administration d'anticorps monoclonaux anti-

* Laboratoire de Parasitologie, Faculté de Médecine, 15 rue de l'École de Médecine, 75270 Paris Cedex 06, France. Tél : 43296525 - Fax : 43295192 .

** Adresse actuelle : Laboratoire de Parasitologie-Mycologie, Hôpital Kremlin-Bicêtre.

Correspondance: F. Derouin, Laboratoire de Parasitologie, Faculté de Médecine, 15 rue de l'Ecole de Médecine, 75270 Paris Cedex 06, France. - Tél : 43296525 - Fax : 43295192. 
in a dissemination of infection; however, using these models it could be clearly demonstrated that immunity plays a major adjunctive role in the control of acute infection.

Concurrent infections between viruses and parasites is a common feature in immunocompromised patients and especially during AIDS. Experimental models of dual infections were developed in an attempt to explain the pathogeny of $T$. gondii in hosts presenting virus induced immunodeficiencies. Mice co-infected with $T$. gondii and the retrovirus LP-BM5, responsible for the Murine Acquired Immunodeficiency Syndrome (MAIDS), and cats co-infected with T. gondii and the Feline Immunodeficiency Virus (FIV) are more sus ceptible to primary acquired toxoplasmosis, but reactivation of chronic infection is unconstantly observed. These studies also showed that Toxoplasma infection also interacts with the natural course of MAIDS. Other experimental models of concurrent infections in which T. gondii was associated with other opportunistic pathogens were developed. Coinfection with Pneumocystis carinii and T. gondii has been realized in immunocompromised rats and this model was used for assessing the efficacy of combined prophylaxis against both pathogens. These models are certainly more difficult to realize and to standardize but are intended to closely simulate the features of the human diseases and to provide a better understanding of the complex interactions between pathogens and host defense

KEY WORDS : toxoplasmosis. Toxoplasma gondii. experimental models. pharmacology.

MOTS CLÉS : toxoplasmose. Toxoplasma gondii. modèles expérimentaux. pharmacologie. lymphocytes T CD4 et CD8 ou anti-interféron gamma. Une autre approche expérimentale consiste à infecter des animaux génétiquement immunodéficients : ces modèles sont peu applicables en pharmacologie car l'infection est généralement disséminée, mais ils ont apporté la preuve du rôle complémentaire majeur de l'immunité dans le traitement de la toxoplasmose aiguë.

L'interaction entre infection virale et parasitaire est la règle chez la plupart des malades immunodéprimés et en particulier au cours du SIDA. Des modèles expérimentaux d'infection mixte ont été mis au point dans le but d'explorer la pathogénicité de T. gondii chez des hôtes immunodéprimés par une infection virale. Des souris co-infectées par T. gondii et le rétro-virus LP-BM5, responsable du SIDA murin, ou des chats co-infectés par T. gondii et le "Feline Immunodeficiency Virus" (FIV) ont une sensibilité accrue à la toxoplasmose acquise mais la réactivation d'une infection chronique n'est pas constamment obtenue. Ces études ont également montré que l'infection parasitaire exerçait un effet inhibiteur sur l'infection virale par le virus LP-BM5. D'autres modèles expérimentaux ont été réalisés, associant $T$. gondii à d'autres pathogènes opportunistes. Un modèle d'infection mixte, associant T. gondii et Pneumocystis carinii, a été réalisé chez le rat; il a été utilisé en particulier pour démontrer l'efficacité de prophylaxies combinées de la toxoplasmose et de la pneumocystose. Ces modèles ont certainement l'inconvénient d'être difficiles à réaliser et à standardiser mais ils représentent une meilleure approche des infections humaines et devraient permettre de mieux appréhender l'étude des mécanismes complexes qui régissent les relations hôte-parasite en cas d'infections multiples.
The real value of any animal model used depends less on the excellence of the model itself than on the ability of the investigators to interpret the results in full awareness of not only the merits, but also the limitations of the experimental system.

O. ZAK, T. O'ReILLY. Animal models as predictors of the safety and efficacy of antibiotics. European Journal of Clinical Microbiology and Infectious Diseases, 1990, 9, 472-478

L a toxoplasmose est actuellement une infection opportuniste fréquente chez les patients atteints de SIDA ou présentant une immunodéficience sévère et reste également une cause importante de foetopathie consécutive à une contamination maternelle en cours de grossesse.

De nombreux modèles expérimentaux ont été proposés pour étudier la physiopathologie de l'infection mais aussi pour évaluer l'efficacité des médicaments dans le traitement ou la prévention de la toxoplasmose. Dans cette revue, nous décrirons ces modèles en insistant tout particulièrement sur ceux qui ont fait, ou qui peuvent faire, l'objet d'applications pharmacologiques.

\section{GÉNÉRALITÉS SUR LES MODÈLES ANIMAUX DE TOXOPLASMOSE ACQUISE}

$\mathrm{D}$ e nombreux animaux peuvent être utilisés du fait de l'ubiquité du parasite, mais l'infection résultante peut revêtir des formes totalement différentes, en fonction de l'espèce animale utilisée (Mc Leod et al., 1989), mais surtout aussi de la souche de toxoplasme, de son mode d'inoculation, de la nature et de la taille de l'inoculum (Johnson, 1984). Actuellement, malgré les apports récents de l'enzymologie et de la biologie moléculaire dans la classification des souches de toxoplasme (Dardé et al., 1992; Howe et al., 1994; Cristina et al., 1995), les critères de différentiation des souches restent encore assez peu précis et il est encore habituel de les classer comme "virulentes ", "peu virulentes " ou " non virulentes ". L'infection résultante peut être suraiguë avec une mortalité de $100 \%$ en cinq à sept jours avec des souches de haute virulence (souche RH) ou réaliser des formes dans lesquelles l'infection est plus progressive réalisant en quelques semaines à quelques mois un tableau de toxoplasmose disséminée avec prédominance de l'atteinte cérébrale. Enfin dans certains cas, l'infection reste complètement latente et ne 


\begin{tabular}{|c|c|}
\hline $\begin{array}{r}\text { Animaux } \\
\mathbf{u} \\
- \\
- \\
-\end{array}$ & $\begin{array}{l}\text { utilisés : } \\
\text { - rongeurs (souris, rat, lapin, cobaye, hamster) } \\
\text { - mouton, porc } \\
\text { - primates }\end{array}$ \\
\hline Souches de & $\begin{array}{l}\text { de } \boldsymbol{T} \text {. gondii : mal définies } \\
\text { - classées comme "virulentes ", "peu virulentes ", "non virulentes" }\end{array}$ \\
\hline $\begin{array}{r}\text { Infection } \\
- \\
-\end{array}$ & $\begin{array}{l}\text { - PO par gavage avec des kystes provenant de cerveaux de souris infectées ou des oocystes } \\
\text { - IP, IM, IV : tachyzoïtes, kystes purifiés, bradyzoïtes }\end{array}$ \\
\hline Résultats : & $\begin{array}{l}\text { : } 0 \text { à } 100 \% \text { de mortalité, en fonction de l'hôte, de la souche de } T \text {. gondii, de la taille de l'inoculum et du stade parasitaire } \\
\text { inoculé }\end{array}$ \\
\hline
\end{tabular}

Tableau I. - Modèles animaux de primo-infection

se manifeste que par la présence de parasites kystiques dans le cerveau (tableau I).

Il est à noter que certains animaux ont une résistance " naturelle "importante, notamment le rat qui peut tolérer des inoculums élevés de souches de parasites de grande virulence, en particulier de la souche $\mathrm{RH}$, en produisant une infection chronique avec formation de kystes cérébraux (Lecomte et al., 1992). Un tel modèle expérimental reste d'un intérêt limité pour l'étude expérimentale du traitement des primo-infections mais il s'avère particulièrement utile pour la compréhension des mécanismes de résistance et d'induction de l'immunité (Benedetto et al., 1993; Darcy et al., 1993).

\section{MODALITÉS D'ÉVALUATION DE L'INFECTION PARASITAIRE}

L'intérêt d'un modèle expérimental et la qualité des informations qu'il peut fournir dépendent totalement des critères d'évaluation de l'infection, de leur sensibilité et surtout de leur reproductibilité. Dans la toxoplasmose, plusieurs modalités de suivi peuvent être proposées, qui ont chacune leurs avantages mais aussi leurs limites.

a) L'étude de la survie reste le premier critère à prendre en compte dans une étude expérimentale chez l'animal. Son estimation doit de préférence être réalisée par la méthode de Kaplan Meier, surtout si des animaux doivent être prélevés en cours d'expérimentation. Cette méthode permet ensuite une analyse statistique comparative sur l'ensemble des courbes de survie, ce qui est nettement plus informatif qu'une comparaison des études de pourcentage de survie à un temps donné.

Le critère " survie " n'est évidemment utilisable que lorsque l'infection parasitaire est létale, ce qui n'est pas le cas dans de nombreuses formes de toxoplasmose expérimentale. Certains signes cliniques tels que l'aspect du poil, le comportement, les mouvements anormaux, l'amaigrissement peuvent alors être utilisés comme de bons marqueurs d'évolutivité d'une infection (Stahl et al., 1988).

b) Etudes histopathologiques : elles sont avant tout qualitatives et permettent de décrire les lésions, en relation avec la présence des parasites. Cependant, ce type d'étude reste difficile à réaliser lorsqu'un nombre important d'organes est à examiner chez de nombreux animaux. De plus, la mise en évidence des parasites dans les tissus est souvent difficile et nécessite l'utilisation de techniques d'immunomarquage (Conley et al., 1981); l'utilisation d'anticorps monoclonaux permet éventuellement de caractériser les stades parasitaires impliqués. La microscopie électronique peut apporter des informations importantes, notamment sur les relations parasite-cellule, la morphologie des différents stades parasitaires et notamment la structure des kystes et des parasites intrakystiques (Ferguson et al., 1994a).

c) Le titrage parasitaire est un élément quantitatif essentiel.

Une méthode microscopique simple consiste à compter les kystes de toxoplasme dans plusieurs échantillons calibrés de broyat de cerveau; il est indispensable de multiplier les échantillons chez un même animal pour obtenir une estimation convenable de la charge parasitaire cérébrale. Cette méthode n'est pas applicable pour d'autres organes.

Un titrage plus précis peut être obtenu par subinoculation à l'animal de dilutions de sang ou d'organes infectés. Cette méthode est d'une grande sensibilité mais elle est limitée par le nombre d'échantillons à étudier, sachant que pour chaque échantillon à titrer, quatre ou cinq dilutions doivent être inoculées à un animal neuf.

De plus, l'examen des souris sub-inoculées est fastidieux car il fait appel à la sérologie puis à l'examen 
direct de plusieurs fragments de cerveau, 30 jours après l'inoculation.

L'utilisation des techniques de culture cellulaires en micro-méthode a permis de grandement faciliter la quantification des charges parasitaires dans le sang ou les organes (Piketty et al., 1991). Le sang ou les broyats d'organes (homogénéisés au Potter ou à l'aide d'un broyeur mécanique) sont dilués dans du milieu de culture et un échantillon de chaque dilution est inoculé dans des cultures cellulaires (fibroblastes MRC5) préparées dans des plaques de microtitration. Après 72 heures d'incubation, les cultures sont fixées au méthanol puis séchées. La révélation des parasites se fait par immunofluorescence indirecte et le titre parasitaire est déterminé par la dernière dilution ayant donné au moins un foyer parasitaire en culture : en rapportant le titre au poids de l'organe ou au volume de sang, il est possible d'exprimer la charge parasitaire en nombre de parasites par gramme d'organe ou par millilitre de sang. Cette technique peut également être utilisée pour différencier les stades parasitaires (tachyzoïtes ou bradyzoïtes) en effectuant le titrage à partir de tissu traité ou non traité par la trypsine, le titre obtenu après digestion trypsique correspondant aux bradyzoïtes trypsinorésistants (Derouin et al., 1991).

d) Les techniques de biologie moléculaire, et en particulier la Polymerase Chain Reaction (PCR) commencent à être appliquées à l'étude de la toxoplasmose expérimentale, dans différents modèles (Weiss et al., 1993; Wastling et al., 1993; Joss et al., 1993; Hitt et al., 1992; Paugam et al., 1995). La PCR n'apparaît pas d'une sensibilité supérieure à l'inoculation à l'animal ou la culture cellulaire pour la recherche de toxoplasme dans les tissus de souris infectées (Weiss et al., 1993) mais elle a permis, dans plusieurs modèles expérimentaux, de détecter une parasitémie au cours de la primo-infection, alors que la culture cellulaire restait négative (Paugam et al., 1995).

\section{APPLICATIONS PHARMACOLOGIQUES}

L'étude des survies des animaux infectés puis traités, comparativement aux animaux infectés mais non traités, représente la base de la plupart des études pharmacologiques dans la toxoplasmose, mais elle a de nombreuses limites et ne peut représenter qu'une étape préliminaire de criblage ou de recherche d'activités synergiques entre médicaments.

Les études histopathologiques peuvent être plus informatives, notamment parce qu'elles permettent de décrire les lésions et d'apprécier la réaction de l'hôte, et en particulier l'importance de la réponse inflammatoire. Un exemple est apporté par l'activité de la rifabutine dans la toxoplasmose chronique, où le traitement permet une augmentation de survie, sans réduction du nombre de kystes cérébraux mais avec une diminution remarquable de l'inflammation tissulaire (Araujo et al., 1994). En complément, la microscopie électronique peut être utile, notamment pour mettre en évidence un effet cytopathogène sur les parasites, dû aux médicaments, comme cela a pu être démontré sur les parasites intrakystiques, dans le cerveau de souris traitées par de l'atovaquone (Ferguson et al., 1994b).

Les différentes techniques de titrage, et notamment celles qui reposent sur la culture cellulaire ou la subinoculation à l'animal ont permis de beaucoup mieux connaître la cinétique de l'infection parasitaire chez l'hôte (Dubey et al., 1976; Candolfi 1992, Derouin et al., 1991; Caroff et al., 1994; Sumyuen et al., 1995). La contamination par voie orale conduit à une infection très précoce des plaques de Peyer, puis une atteinte pulmonaire importante, spontanément résolutive après 50 jours; parallèlement, l'atteinte cérébrale se développe puis se stabilise en 30 à 50 jours. Dans les modèles d'infection aiguë (souche $\mathrm{RH}$ par exemple), on observe une augmentation rapide des charges parasitaires pulmonaire, cérébrale et sanguine, associée à une détresse respiratoire et à des troubles encéphaliques (Piketty et al., 1991; Derouin et al., 1991), aboutissant à une mort rapide.

Sur le plan pharmacologique, la connaissance de cette cinétique d'infection dans les tissus a des retombées importantes sur le choix des médicaments en fonction de leurs propriétés pharmacocinétiques. L'étude du suivi des charges parasitaires chez des souris infectées avec une souche virulente puis traitées a clairement montré que les médicaments avaient des mécanismes d'action très différents (Piketty et al., 1991). Un exemple caractéristique est donné par l'azithromycine, macrolide de nouvelle génération, dont l'action antiparasitaire se manifeste presque électivement sur l'infection pulmonaire, alors que les charges parasitaires cérébrales sont croissantes pendant le traitement. Cette action peut s'expliquer en grande partie par le fait que l'azithromycine se concentre remarquablement dans les macrophages alvéolaires, alors qu'elle reste à taux faibles dans le tissu cérébral (Derouin et al., 1992). En conséquence il n'est pas envisageable d'utiliser l'azithromycine (ou tout autre macrolide) en monothérapie dans le traitement des formes cérébrales de toxoplasmose, mais son utilisation dans les formes multiviscérales et pulmonaires est possible, en association avec des médicaments dont la diffusion cérébrale est bonne (tels que la pyriméthamine, la sulfadiazine ou l'atovaquone). Avec ces associations, on observe expérimentalement une remarquable synergie sur les charges tissulaires du fait de la complémentarité d'action des deux médica- 
ments sur les principaux sites infectieux (Derouin $e t$ al., 1992).

Ces techniques de titrage par culture cellulaire ont donc permis de reconsidérer le mode d'action de la plupart des médicaments ou associations de médicaments anti-parasitaires, et de proposer leur utilisation à partir des bases expérimentales fiables, comparables à celles qui sont exigées pour les médicaments antibactériens (Derouin et al., 1993). De plus, la culture ou l'inoculation à l'animal ne quantifient que les parasites viables, ne permettant de distinguer que les médicaments parasitostatiques et parasiticides. Une illustration est apportée par l'étude réalisée par Huskinson-Mark et al. (1991) sur l'activité des médicaments sur des kystes de toxoplasme isolés de cerveaux de souris : l'incubation de ces kystes avec différents médicaments n'a pas montré d'altération morphologique notable; par contre la sub-inoculation à l'animal des kystes incubés avec l'azithromycine ou l'atovaquone était négative, démontrant l'activité de ces médicaments sur les parasites intrakystiques.

Quant aux techniques de biologie moléculaire, leurs applications en pharmacologie expérimentale restent encore à développer, en particulier la PCR quantitative qui pourrait être utilisée pour le suivi de l'efficacité d'un traitement, sans que le sacrifice de l'animal d'expérience soit nécessaire.

\section{TOXOPLASMOSE CONGÉNITALE EXPÉRIMENTALE (tableau Il).}

L a réalisation de modèles animaux de toxoplasmose congénitale se heurte à des difficultés évidentes en raison des différences importantes qui existent dans les durées de gestation chez les animaux, la durée de la parasitémie, et le type de pla- centa qui conditionne la transmission materno-foetale (Darcy et al., 1991; Beverley, 1969; Beverley et al., 1973). De plus, l'évaluation de l'infection foetale reste difficile, notamment avec des petits animaux (souris, rats, cobayes) chez lesquels il n'est pratiquement possible d'évaluer que le pourcentage de transmission materno-foetale, par examen histologique des fœtus ou par sub-inoculation. Ces modèles, sur petits animaux, ont cependant permis de mettre en évidence d'importantes différences de virulence et de transmission suivant les souches utilisées chez le rat (Dubey et al., 1991; Zenner et al., 1993), d'estimer succintement le risque de transmission fotale en fonction de la date de contamination de la souris et du cobaye (van de Waaij et al., 1960; Huldt, 1960) et de réaliser un modèle simple de toxoplasmose oculaire, associant des lésions de cataracte et de chroriorétinite (Hutchison et al., 1982; Hay et al., 1984). Les autres types de lésions anatomiques observées chez les foetus infectés ont surtout été décrites lors d'infections congénitales réalisées sur de plus gros animaux tel le porc (Dubey et al., 1990), le mouton (Buxton et al., 1986; Beverley et al., 1973). Récemment, un modèle expérimental utilisant le singe rhésus a été décrit par Schoondermark-van de Ven et al., (1993), dans lequel le risque de transmission materno-foetale a été évalué à la suite de contaminations maternelles réalisées aux deuxième et troisième trimestres de gestation. Ces auteurs ont réalisé un suivi de l'infection foetale par inoculation à la souris et par PCR sur des prélèvements de liquide amniotique et de sang foetal. La transmission materno-foetale a été obtenue dans 40 à $60 \%$ des cas, mais il n'a pas été observé de parasitémie chez les foetus infectés, ni de signe clinique. Ce modèle, qui reste malgré tout assez proche de l'infection humaine, a également permis de prouver l'efficacité de la spiramycine dans la prévention de la transmission materno-foetale, en montrant notam-

1 - Rongeurs : souris, rat, cobaye :

- 0 à $70 \%$ de transmission

- mort foetale ou toxoplasmose chronique

- peu ou pas d'études pharmacologiques (sauf pour l'évaluation de l'effet tératogène des médicaments)

(Dubey et al., 1976; Huldt 1960; Van Der Waaij 1960; Hutchison et al., 1982; Hay et al., 1984; Zenner et al., 1993)

2-Mouton :

- importantes lésions placentaires. Avortement

- encéphalite chez l'agneau.

(Beverley 1969; Beverley et al., 1973; Buxton et al., 1986)

3 - Primate (singe rhésus) :

- 44-61\% de transmission

- pas de parasitémie chez les foetus infectés

- absence de signes cliniques

- Etudes pharmacologiques : spiramycine, pyriméthamine/sulfadiazine

(Schoondermark-van de Ven et al., 1993, 1994a, 1994b, 1995)

Tableau II. - Modèles animaux de toxoplasmose congénitale 
Toxoplasmose congénitale ou primo-infection chez les petits rongeurs (souris, cobaye) :

- examen anatomopathologique et clinique (biomicroscope)

- Lésions : chroriorétinite, nécrose périvasculaire, cataracte

(Hutchison et al., 1982; Hay et al., 1984; Dutton et al., 1984a, 1984b; Skorich et al., 1988; Mc Menamin 1986; Ollé et al., 1994 Gazzinelli et al., 1994)

Primo-infection chez le chat :

- injection de tachyzoïtes dans la carotide

- examen anatomopathologique et ophtalmoscopique

- Lésions : chroriorétinite granulomateuse

(Davidson et al., 1993)

\section{Primo-infection chez le lapin :}

- injection de tachyzoïtes dans l'espace suprachoroïdal

- examen anatomopathologique et ophtalmoscopique

- choriorétinite régressant spontanément

(Tabbara et al., 1974; Rollins et al., 1982; Nozik et al., 1968)

\section{Primo-infection chez les primates :}

- injection intra-rétinienne de tachyzoïtes

- examen anatomopathologique et ophtalmoscopique

- choriorétinite puis cicatrice pigmentée

- pas de réactivation après traitement immunosuppresseur.

(Newman et al., 1982; Holland et al., 1988; Culberston et al., 1982)

Tableau III. - Modèles animaux de toxoplasmose oculaire

\begin{tabular}{|c|c|}
\hline Type d'infection & Manifestations pathologiques \\
\hline $\begin{array}{l}\text { Toxoplasmose aiguë } \\
\text { infection IP, souche RH } \\
\text { (Piketty et al., 1990; Weiss et al., 1991) }\end{array}$ & $\begin{array}{l}\text { Survie : 5-7 jours. Toxoplasmose disséminée. } \\
\text { Prédominance de l'atteinte pulmonaire, cérébrale. } \\
\text { Parasitémie importante. }\end{array}$ \\
\hline $\begin{array}{l}\text { Toxoplasmose subaiguë } \\
\text { infection IP ou PO } \\
\text { (Candolfi 1992; Dumas et al., 1994) }\end{array}$ & $\begin{array}{l}\text { Survie : } 60-150 \text { jours. } \\
\text { Lésions dégénératives progressives avec signes neurologiques. }\end{array}$ \\
\hline $\begin{array}{l}\text { Toxoplasmose chronique } \\
\text { infection PO } \\
\text { (Stahl et al., 1988; Derouin et al., 1991) }\end{array}$ & $\begin{array}{l}100 \text { \% survie. Asymptomatique. } \\
\text { Atteinte pulmonaire spontanément résolutive. } \\
\text { Charges parasitaires cérébrales stables après } 90 \text { jours d'infection. }\end{array}$ \\
\hline $\begin{array}{l}\text { Encéphalite toxoplasmique: } \\
\text { focale. } \\
\text { Infection intra-cérébrale. } \\
\text { (Hofflin et al., 1987a, 1987b, 1987c) }\end{array}$ & $\begin{array}{l}\text { 100\% de mortalité si l'infection est associé à un traitement par les } \\
\text { corticoïdes. } \\
\text { Abcès cérébraux focalisés. }\end{array}$ \\
\hline
\end{tabular}

Tableau IV. - Modèles murins d'encéphalite toxoplasmique. 1 - Primo-infection chez un hôte non immunodéprimé

ment, une forte concentration dans le tissu placentaire (Schoondermark-van de Ven et al., 1994a-b), et un effet stérilisateur sur le foetus lors du traitement maternel par l'association pyriméthamine-sulfadiazine (Schoondermark-van de Ven et al., 1995).

\section{TOXOPLASMOSE OCULAIRE EXPÉRIMENTALE (tableau III)}

es modèles animaux de toxoplasmose oculaire ont surtout été développés pour comprendre la physiopathologie de la choriorétinite toxoplas- mique, dont on peut discuter les origines strictement parasitaire et/ou auto-immune. Les différents modèles proposés se sont heurtés à deux obstacles majeurs :

a) la réalisation d'une infection oculaire focalisée : dans la plupart des cas, il s'agit d'une inoculation in situ dont le caractère traumatique peut modifier l'évolution naturelle de la maladie;

b) l'évaluation clinique et biologique de l'infection oculaire : elle est rendue particulièrement difficile du fait de la taille des yeux chez les petits animaux et de la grande difficulté de quantifier les charges parasitaires oculaires. Différentes techniques ont été proposées pour l'examen ophtalmologique de la rétine 
chez des animaux de petite taille (Dutton et al., 1984a; Ollé et al., 1994) mais le suivi clinique des lésions reste difficile. Seuls les modèles réalisés chez des animaux comme le lapin (Nozik et al., 1968; Rollins et al., 1982; Tabbara et al., 1974), les primates (Newman et al., 1982 Culberston et al., 1982 ; Holland et al., 1988) ou le chat (Davidson et al., 1993) permettent d'effectuer un suivi ophtalmologique satisfaisant.

La plupart des études réalisées chez les petits rongeurs (souris, cobaye) se sont appuyées sur les techniques histologiques et de microscopie électronique, avec pour objectif de décrire les aspects anatomopathologiques des lésions (Hutchison et al., 1982; Hay et al., 1984; Mc Menamin et al., 1986; Dutton et al., 1984b Skorich et al., 1988). Plus récemment, les techniques de titrage parasitaire par culture ou de recherche d'ADN parasitaire par PCR ont été utilisées (Gazzinelli et al., 1994; Ollé et al., 1994), mais avec peu d'applications en pharmacologie expérimentale.

Le lapin reste l'animal d'expérience le plus utilisé pour l'évaluation des traitements de la toxoplasmose oculaire (Nozik et al., 1968, Rollins et al., 1982, Tabbara et al., 1974) ; l'infection est réalisée de façon artificielle par injection de tachyzoïtes dans l'espace suprachoroïdal mais les lésions obtenues sont assez semblables à celles observées chez l'homme. Ce modèle a permis de montrer l'efficacité de la clindamycine et de la minocycline dans la toxoplasmose oculaire (Rollins et al., 1982; Tabbara et al., 1974), mais l'extrapolation à l'homme reste difficile, du fait de notre méconnaissance de la pharmacocinétique de ces médicaments dans l'oeil, aussi bien chez l'animal que chez l'homme.

Les modèles expérimentaux de toxoplasmose oculaire réalisés chez les primates ont apporté peu d'informations complémentaires. Les résultats obtenus par différentes équipes restent difficiles à interpréter et à extrapoler à l'homme car les modalités d'infection sont traumatiques (injection intra-oculaire) et l'évolution des lésions est différente de celle qui est observée en cas de toxoplasmose congénitale ou d'immunodépression chez l'homme (Newman et al., 1982; Culberston et al., 1982; Holland et al., 1988).

\section{TOXOPLASMOSE CÉRÉBRALE EXPÉRIMENTALE (tableaux IV-VII)}

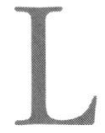

a fréquence croissante des cas de toxoplasmose cérébrale chez les malades immunodéprimés, en particulier au cours du SIDA, a largement contribué à stimuler la recherche expérimentale dans la toxoplasmose. Différents modèles animaux d'encéphalite toxoplasmique ont été développés pour identifier les mécanismes de réactivation et rechercher des nouvelles thérapeutiques ou prophylaxies. On peut tenter de regrouper ces différents modèles en trois catégories bien distinctes, dont les modalités expérimentales, les objectifs et les informations recueillies sont très différentes.

\section{LES MODÈLES “ GÉNÉRALISTES " (tableau IV)}

Ce sont les plus couramment utilisés :

- toxoplasmose aiguë au cours de laquelle l'atteinte cérébrale est associée à une atteinte multi-viscérale,

- toxoplasmose subaiguë dans laquelle des lésions dégénératives sont observées avec apparition progressive de signes neurologiques,

- toxoplasmose chronique au cours de laquelle on n'observe aucune mortalité mais une localisation cérébrale des kystes.

Dans chaque catégorie de modèle, les résultats vont dépendre de la virulence de la souche, de la taille de l'inoculum, du type de souris utilisé, et de grandes variations sont observées entre les laboratoires, du fait de ces conditions expérimentales différentes. Ces modèles ont l'avantage d'une relative simplicité et ils ont été largement utilisés pour l'évaluation expérimentale des médicaments anti-parasitaires : les modèles d'infection aiguë, associés à une mortalité de $100 \%$ en 8 à 10 jours seront préférés pour les études de traitement, du fait de leur bonne reproductibilité; les modèles d'infection subaiguë ou chronique seront préférés pour les études de prophylaxie ou celles visant à évaluer l'efficacité des médicaments sur les formes kystiques (Dumas et al., 1994; Ferguson et al., 1994b; Araujo et al., 1992).

Comme nous l'avons mentionné précédemment à propos des modèles de primo-infection, le critère "survie " reste utile, mais il est peu informatif quant à l'action des médicaments sur l'infection cérébrale. Les études par comptage des kystes, histologie et titrage parasitaire sont donc indispensables. Le titrage des parasites cérébraux apporte une information essentielle sur l'importance mais aussi sur la rapidité d'action des médicaments sur les parasites cérébraux. Cependant, l'extrapolation à l'homme des résultats observés chez l'animal reste difficile pour deux raisons : a) l'infection obtenue dans ces modèles est une toxoplasmose généralisée, souvent sévère, au cours de laquelle l'atteinte cérébrale n'est qu'une des composantes de l'infection; il s'agit donc d'une situation assez différente de celle que l'on observe chez les malades immunodéprimés, chez qui l'infection est le plus souvent focalisée, b) la pharmacocinétique et le métabolisme des médicaments sont différents chez l'animal et chez l'homme et il n'existe pas de "règle" 
permettant de transposer directement les doses trouvées efficaces chez l'animal à un schéma thérapeutique strictement comparable chez l'homme (Craig 1990). L'interprétation des résultats obtenus doit donc être prudente et tenir compte de ces limites (Zak et al., 1990).

\section{UN MODÈLE “ SPÉCIFIQUE " (tableau IV)}

Chez les sujets immunodéprimés, la toxoplasmose se présente avant tout comme une encéphalite focalisée. Dans le but d'obtenir un modèle expérimental plus proche de la situation clinique, Hofflin et al. (1987a) ont proposé de réaliser une infection cérébrale in situ, par injection de tachyzoïtes dans le lobe frontal de la souris. Il en résulte la formation de foyers inflammatoires puis nécrotiques, riches en parasites, évoluant secondairement vers une toxoplasmose disséminée lorsque les animaux reçoivent un traitement immunosuppresseur. Ce modèle présente l'avantage de produire des lésions ayant une grande similitude avec celles observées chez l'homme, mais il est de réalisation très délicate et n'a été maîtrisé que dans un seul laboratoire. Ses applications pharmacologiques sont restées limitées à l'étude de la clindamycine, de la roxithromycine et de l'interféron gamma (Hofflin et al., 1987 b-c).

\section{LES MODÈLES ASSOCIÉS À UNE IMMUNODÉPRESSION}

Tous ces modèles ont comme objectif de réaliser une infection toxoplasmique dans des conditions de survenue comparables à celles observées chez les malades immunodéprimés. Plusieurs approches ont été développées, réalisant des modèles complexes, chez des animaux totalement ou partiellement immunodéprimés.

a) Administration de médicaments immunosuppresseurs (tableau V)

L'effet de médicaments immunosuppresseurs sur l'évolution de la toxoplasmose a fait l'objet de nom- breuses études expérimentales mais les résultats obtenus sont différents suivant les modèles utilisés. Chez le hamster, Frenkel et al. (1975) ont pu obtenir une dissémination parasitaire à la suite d'une primo infection et une réactivation d'une infection chronique par administration de corticoïdes, associée ou non à une irradiation corporelle. Dans ces cas, les lésions étaient prédominantes dans les poumons, le cerveau et l'oeil. Chez la souris, Stahl et al. (1966) ont obtenu des méningo-encéphalites diffuses après traitement par de la cortisone, l'irradiation ou la splénectomie. Plus récemment, Candolfi (1992) a étudié l'effet de la ciclosporine et de l'hydrocortisone chez des souris infectées par une souche peu virulente; il n'a observé de réactivation que lors du traitement par la ciclosporine, sans aggravation des lésions anatomopathologiques, mais avec une parasitémie, une antigénémie et une modification de la cinétique des anticorps sériques.

Chez le rat, l'administration d'immunosuppresseurs et en particulier de corticoïdes permet d'atténuer la résistance naturelle (Benedetto et al., 1993) et d'obtenir une infection létale avec prédominance au niveau du foie, de la rate et des poumons, fréquemment accompagnée d'un épanchement pleural riche en parasites (Brun-Pascaud et al., 1994).

Dans notre expérience, chez la souris infectée par une souche peu virulente, le traitement par corticoïdes, azathioprine et ciclosporine utilisés seuls ou en associations n'entraîne pas d'augmentation importante des charges parasitaires, sauf au niveau pulmonaire lorsque le traitement est administré précocement au cours de la primo-infection ( $\mathrm{Su}-$ myuen, 1995). Au total, ces modèles sont de maniement difficile car ils reposent sur l'utilisation de médicaments dont les effets immunosuppresseurs sont complexes et pas toujours élucidés. Ces modèles apportent finalement peu d'informations sur les mécanismes de réactivation cérébrale et n'ont que peu d'applications pharmacologiques directes.

\begin{tabular}{|c|c|}
\hline Modèles & Manifestations pathologiques \\
\hline $\begin{array}{l}\text { Hamster traité par corticoïdes } \\
\text { (Frenkel et al., 1975) }\end{array}$ & Encéphalite toxoplasmique et pneumopath \\
\hline $\begin{array}{l}\text { Souris traitée par immunosuppresseur } \\
\text { (corticoïdes, azathioprine) } \\
\text { (Stahl et al., 1966; Candolfi 1992; Sumyuen et al., non publié) }\end{array}$ & $\begin{array}{l}\text { Pas d'augmentation de mortalité. Charges } p \\
\text { augmentées si le traitement est administre } \\
\text { infection }\end{array}$ \\
\hline $\begin{array}{l}\text { Souris traitée par anti-CD4 } \\
\text { anti-CD8 ou anti-IFN } \gamma\end{array}$ & Réactivation (cerveau). 30-100\% mortalité \\
\hline
\end{tabular}

Tableau V. - Modèles animaux d'encéphalite toxoplasmique. 2 - Réactivation de toxoplasmose chez un hôte immunodéprimé 
b) Modification de la réponse immunitaire spécifique par administration d'anticorps dirigés contre des lymphocytes ou des cytokines (tableau V)

Cette approche expérimentale a surtout été développée pour identifier le ou les facteurs directement impliqués dans les mécanismes de réactivation. Les résultats les plus démonstratifs ont été obtenus par l'utilisation d'anticorps monoclonaux ou polyclonaux anti-IFN- $\gamma$, permettant d'obtenir une dissémination au cours d'une primo-infection par une souche de faible virulence, une réactivation cérébrale focalisée (Suzuki et al., 1988; Suzuki et al., 1990), une choriorétinite (Ollé et al., 1994) et une augmentation significative des charges parasitaires pulmonaires et cérébrales (Sumyuen, 1995). L'utilisation d'anticorps anti-lymphocytes T CD4 ou CD8 a permis de préciser le rôle respectif de ces sous-populations cellulaires dans le contrôle de l'infection chronique, mais il n'a été possible d'obtenir des réactivations qu'après des traitements combinés par anti-CD4 et anti-CD8, éventuellement en association avec un anticorps antiIFN- $\gamma$ (Gazzinelli et al., 1992).

Ces modèles expérimentaux sont certainement plus proches de la réalité pathologique que ceux qui utilisent des médicaments immunosuppresseurs, mais ils ont l'inconvénient d'être difficiles à réaliser, très coûteux et peu applicables aux études pharmacologiques qui nécessitent souvent un grand nombre d'animaux.

\section{c) Utilisation d'animaux génétiquement immunodéfi- cients (tableau VI)}

L'utilisation de souris ou de rats "nude " et de souris SCID permet d'obtenir des formes graves de toxoplasmose à la suite de primo-infections par des souches de faible virulence (Johnson, 1992; Beaman et al., 1994; Foulet et al., 1994). Ces modèles ont démontré le rôle prédominant de l'immunité cellulaire et de ses différentes composantes dans le contrôle de la phase aiguë de la toxoplasmose. Sur le plan pharmacolo- gique, ils ont permis de démontrer le rôle déterminant de l'immunité cellulaire dans l'efficacité d'un traitement de toxoplasmose aiguë (Murray et al., 1993).

\section{d) Co-infections avec des virus induisant une immu- nodépression (tableau VII)}

Ces modèles ont avant tout pour objectif d'étudier expérimentalement les réactivations toxoplasmiques associées à une infection virale, telles qu'elles sont observées chez l'homme lors d'infection par le cytomégalovirus et surtout par le Virus de l'Immunodéficience Humaine (VIH).

Expérimentalement, de nombreux virus peuvent induire une immunodépression parmi lesquels le virus de Friend, le virus de la chorioméningite lymphocytaire, le cytomegalovirus murin (CMVM) et les rétrovirus : lentivirus pour le "Feline Immunodeficiency Virus" (FIV) et le "Simian Immunodeficiency Virus" (SIV), oncovirus pour le complexe viral LPBM5 MuLV responsable du "SIDA murin".

La réactivation d'une toxoplasmose chronique n'a $\mathrm{pu}$ être observée que dans les modèles utilisant le CMVM (Pomeroy et al., 1992). Chez la souris préalablement infectée par Toxoplasma gondii, l'inoculation du CMVM induit une réactivation qui se manifeste essentiellement sous la forme d'une pneumonie avec afflux local de lymphocytes T CD8+.

Chez le chat, la réactivation d'une toxoplasmose chronique préexistante n'a jamais pu être réalisée par la co-infection avec le FIV. A l'inverse, l'infection virale favorise l'installation du parasite, avec une moins bonne régulation de l'infection parasitaire. Dans un des modèles (Davidson et al., 1993), ceci peut entraîner une toxoplasmose disséminée se manifestant particulièrement sous la forme d'une pneumopathie interstitielle avec $75 \%$ de mortalité chez les chats co-infectés; dans l'autre modèle, réalisé par Lin

\begin{tabular}{|ll|}
\hline Modèles & Manifestations pathologiques \\
\hline Souris ou rat nude & $\begin{array}{l}\text { Toxoplasmose disséminée. } \\
100 \% \text { de mortalité (5-7 jours) }\end{array}$ \\
$\begin{array}{l}\text { Souris nude. Traitement par sulfadiazine } \\
\text { pendant } 3 \text { semaines } \\
\text { (Murray et al., 1993, Foulet et al., 1994, Schluter et al., 1991) }\end{array}$ & $\begin{array}{l}\text { Toxoplasmose disséminée. } \\
100 \% \text { de mortalité après un traitement par la sulfadiazine }\end{array}$ \\
\hline $\begin{array}{l}\text { Souris SCID } \\
\text { Souris SCID. Traitement par sulfadiazine } \\
\text { pendant } 3 \text { semaines } \\
\text { (Johnson 1992; Beaman et al., 1994) }\end{array}$ & $\begin{array}{l}\text { Toxoplasmose disséminée. } \\
100 \% \text { de mortalité (5-7 jours) }\end{array}$ \\
\hline
\end{tabular}

Tableau VI. - Modèles murins d'encéphalite toxoplasmique. 3 - Primo-infection d’un hôte génétiquement immunodéficient 


\begin{tabular}{|c|c|c|c|c|}
\hline Modèles & Animal & $\begin{array}{l}\text { Souche de } \\
\text { T.gondii }\end{array}$ & $\begin{array}{l}\text { Souche } \\
\text { virale }\end{array}$ & $\begin{array}{l}\text { Manifestations } \\
\text { pathologiques }\end{array}$ \\
\hline \multicolumn{5}{|c|}{ Cytomégalovirus (MCMV) } \\
\hline \multirow[t]{2}{*}{ Pomeroy et al., 1992} & Souris & $\mathrm{C} 56$ & Souche Smith & \multirow[t]{2}{*}{ Réactivation avec pneumopathie } \\
\hline & $\mathrm{Balb} / \mathrm{c}$ & $6.10^{4}$ tach. IP & $5.10^{4}$ PFU IP & \\
\hline \multicolumn{5}{|c|}{ Feline Immuodeficiency Virus (FIV) } \\
\hline \multirow[t]{2}{*}{ Lappin et al., 1993} & Chats & ME 49 & Petaluma & \multirow[b]{2}{*}{ Absence de réactivation } \\
\hline & Germ free & $10^{3}$ kystes PO & $0,5 \mathrm{ml} \mathrm{sang}$ & \\
\hline \multirow[t]{2}{*}{ Lin et al., 1992} & Chatons & T264 & Augusta & \multirow{4}{*}{$\begin{array}{l}\text { Augmentation des charges } \\
\text { parasitaires (cerveau et ganglions) au } \\
\text { cours de la primo-infection } \\
\text { Toxoplasmose disséminée } \\
\text { (primo-infection) }\end{array}$} \\
\hline & Germ free & $10^{3}$ kystes $\mathrm{PO}$ & $2 \mathrm{ml}$ plasma & \\
\hline Davidson et al., 1993 & Chats & ME 49 & FIV-NCSU1 & \\
\hline & Germ free & $10^{4}$ tach. & $6.10^{5} \mathrm{TCID}_{50}$ & \\
\hline \multicolumn{5}{|l|}{ LP-BM5 (MAIDS) } \\
\hline Gazzinelli et al., 1992 & $\begin{array}{l}\text { Souris } \\
\text { B6 }\end{array}$ & $\begin{array}{l}\text { C56 } 10^{5} \text { tach. IP } \\
\text { ME } 4920 \text { kystes IP }\end{array}$ & $10^{3}$ PFU IP & $\begin{array}{l}\text { Réactivation partielle cérébrale (30\% } \\
\text { de mortalité). Diminution IFN- } \gamma \text {. }\end{array}$ \\
\hline Watanabe et al., 1993 & $\begin{array}{l}\text { Souris } \\
\text { C57Bl/6 }\end{array}$ & $\begin{array}{l}\text { Fukaya } \\
10 \text { kystes IP }\end{array}$ & $3.810^{4}$ PFU IP & $\begin{array}{l}\text { Réactivation cérébrale } \\
\text { (100\% des cas) }\end{array}$ \\
\hline Lacroix et al., 1994 & $\begin{array}{l}\text { Souris } \\
\mathrm{C} 57 \mathrm{Bl} / 6\end{array}$ & $\begin{array}{l}\text { C } \\
10 \text { kystes IP }\end{array}$ & $4.10^{3}$ PFU IP & $\begin{array}{l}\text { Réactivation pulmonaire au cours des } \\
\text { primo-infections } \\
\text { Pas de réactivation d'infection chro- } \\
\text { nique }\end{array}$ \\
\hline
\end{tabular}

PO : per os; IP : intrapéritonéal; PFU : Plaque Forming Units; TCID50 : 50 \% Tissue Culture Infecting Dose; tach : tachyzoïtes.

Tableau VII . - Modèles animaux d'encéphalite toxoplasmique. 4 - Co-infection avec un virus immunodépresseur

et al. (1993), l'augmentation de susceptibilité des animaux vis-à-vis du toxoplasme se manifeste uniquement par une aggravation des signes cliniques.

Chez la souris, les résultats obtenus avec le complexe viral LP-BM5 divergent, en fonction des protocoles expérimentaux utilisés : souches de T. gondii, inoculum viraux et parasitaires, délais entre les deux infections, etc... (Gazzinelli et al.,, 1992; Watanabe et al., 1993; Lacroix et al., 1994). Bien que Watanabe et al. (1993) aient réussi à obtenir une réactivation toxoplasmique cérébrale chez $100 \%$ des souris co-infectées, l'équipe de Gazzinelli et al. (1992) n'obtient qu'une réactivation cérébrale partielle avec seulement 30 à $40 \%$ de mortalité, malgré une diminution de la sécrétion d'IFN- $\gamma$ chez les animaux co-infectés; seule l'addition d'anticorps anti-CD8 ou anti-IFN- $\gamma$ permet d'obtenir une réactivation totale. De même, Lacroix et al. (1994) n'ont observé qu'une faible augmentation des charges parasitaires pulmonaires chez les souris co-infectées sans différence de mortalité par rapport à celles infectées uniquement par T. gondii ou LP-BM5. Les modèles de co-infections inverses (infection par LP-BM5 puis infection toxoplasmique) (Gazzinelli et al., 1992; Lacroix et al., 1994) ont, par ailleurs, permis de mettre en évidence une augmentation de la susceptibilité des souris infectées par le virus vis-à-vis de l'infection parasitaire, avec une moins bonne régulation de l'infection toxoplasmique. Les manifestations cliniques de cette dysrégulation sont essentiellement une atteinte multiviscérale et/ou pulmonaire, confirmant le rôle essentiel joué par le poumon à la phase aiguë de la toxoplasmose.

La co-infection par $T$. gondii et le Simian Immunodeficiency Virus (SIV) n’a jamais été réalisée expérimentalement; on peut juste rapporter deux cas de réactivation toxoplasmique observés chez le macaque après infection par le SIV (Sasseville et al., 1995).

Ces modèles animaux de co-infection avec un virus immunodépresseur et $T$. gondii ont montré également que l'infection parasitaire pouvait modifier la cinétique de l'infection virale. Dans deux études de coinfection réalisées avec le virus LP-BM5, il a été noté que la progression de la rétrovirose (estimée par le poids des rates et des ganglions, marqueurs de la lymphoprolifération) était très diminuée chez les souris co-infectées par $T$. gondii, comparativement aux souris infectées par le virus seul (Gazzinelli et al., 1992; Lacroix et al., 1994). Ces modèles ont donc un réel intérêt pour l'étude des interactions pouvant exister in vivo entre les deux infections. Ils restent cependant de réalisation complexe et encore très éloignés de la co-infection humaine par le VIH et $T$. gondii. Leurs applications pharmacologiques sont potentiellement importantes car ces modèles devraient permettre d'évaluer non seulement l'effet des traitements anti-parasitaires, comme cela a été réalisé pour la 
clindamycine dans le traitement des réactivations induites par le CMVM (Filice et al., 1991), mais aussi l'effet des traitements anti-viraux sur la survenue des réactivations parasitaires (Banister et al., 1993).

\section{MODÈLES COMPLEXES D'INFECTIONS MULTIPLES. PERSPECTIVES}

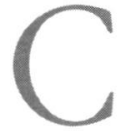

hez les malades immunodéprimés, la toxoplasmose est souvent associée à d'autres infections opportunistes, souvent latentes, dont il faut également prévenir la réactivation ou l'expression pathologique. C'est essentiellement le cas de la pneumocystose, des parasitoses digestives (cryptosporidiose, microsporidiose), et des infections à mycobactéries atypiques. Des modèles de co-infection associant la toxoplasmose et une autre infection parasitaire ont donc été développés dans le but d'évaluer l'activité thérapeutique ou prophylactique des médicaments sur une ou plusieurs infections opportunistes. Un modèle d'infection mixte a été réalisé chez le rat immunodéprimé, permettant d'obtenir une pneumopathie sévère à Pneumocystis carinii, associée à une atteinte multiviscérale à Toxoplasma gondii (Brun-Pascaud et al., 1994). Dans ce modèle, l'infection naturelle à $P$. carinii est réactivée par un traitement avec des corticoïdes puis la toxoplasmose est ensuite associée, par injection de tachyzoïtes d'une souche virulente (RH). Ce modèle d'infections associées, réalisé chez un hôte immunodéprimé, a permis de confirmer l'intérêt de l'association triméthoprime + sulfaméthoxazole et de l'association pyriméthamine + dapsone dans la prévention mixte de ces deux parasitoses.

L'extension de ces modèles complexes à l'étude d'autres infections opportunistes associées, parasitaires ou non (mycobactéries atypiques en particulier) est en plein développement. Des premiers résultats obtenus lors d'une co-infection chronique par T. gondii et Mycobacterium avium ont apporté la preuve qu'il était possible d'envisager des prophylaxies combinées, anti-parasitaires et anti-bactériennes (Maslo et al., 1995).

Cette approche expérimentale représente une voie de recherche tout à fait prometteuse dans la mesure où elle permet d'évaluer expérimentalement des protocoles thérapeutiques aux retombées cliniques évidentes, mais aussi d'aborder les interactions complexes existant chez l'hôte infecté simultanément par plusieurs pathogènes.

En conclusion, la toxoplasmose offre de nombreuses possibilités d'étude expérimentale, du fait du poly- morphisme de cette parasitose. Les applications pharmacologiques de ces différents modèles ont été largement développées ces dernières années mais elles restent encore insuffisantes notamment pour la toxoplasmose congénitale et oculaire. Parallèlement, la réalisation de modèles plus proches de l'infection humaine, ainsi que l'amélioration des méthodes d'investigation chez l'animal doivent également avoir pour objectif de favoriser les études physiopathologiques qui font encore cruellement défaut en matière de toxoplasmose.

\section{REMERCIEMENTS}

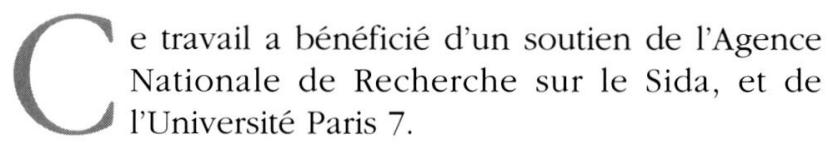

\section{RÉFÉRENCES}

Araujo F.G., Huskinson-Mark J., Gutteridge W.E. \& Remington J.S. In vitro and in vivo activities of the hydroxynaphthoquinone $566 \mathrm{C} 80$ against the cyst form of Toxoplasma gondii. Antimicrobial Agents and Chemotherapy, 1992, 36, 326-330.

Araujo F.G., Slifer T. \& Remington J.S. Rifabutin is active in murine models of toxoplasmosis. Antimicrobial Agents and Chemotherapy, 1994, 38, 570-575.

BANISTER S. \& POMEROY C. Effect of Gancyclovir on murine cytomegalovirus-induced reactivation of Toxoplasma pneumonia. Journal of Laboratory and Clinical Medicine, 1993, 122, 576-580.

Beaman M.H., Araujo F.G. \& Remington J.S. Protective reconstitution of the SCID mouse against reactivation of toxoplasmic encephalitis. Journal of Infectious Diseases, 1994, 169, 375-383.

Benedetto N., Folgore A. \& Galdiero M. Impairment of natural resistance to Toxoplasma gondii infection in rats treated with adrenergics, $\beta$-blockers, $\beta$-corticosteroids or total body irradiation. Pathological Biology, 1993, 41, 404-409.

Beverley J.K.A. Congenital Toxoplasma infections in animals other than man. Colloque sur la toxoplasmose de la femme enceinte, Monographie du "Lyon Médical ". Edit. Specia. 1969, 5-20.

Beverley J.K.A. \& Freeman A.P. Prevention of pathological changes in experimental congenital Toxoplasma infections. Lyon Médical, 1973, 230, 491-498.

Brun-Pascaud M., Chau F., Simonpoli A.M., Girard P.M., Derouin F. \& Pocidalo J.J. Experimental evaluation of combined prophylaxis against murine pneumocystis and toxoplasmosis. Journal of Infectious Diseases, 1994, 170, 653-658.

BuxTON D. \& Finlayson J. Experimental infection of pregnant sheep with Toxoplasma gondii: pathological and 
immunological observations on the placenta and foetus. Journal of Comparative Pathology, 1986, 96, 319-333.

CANDOlfi E. Les marqueurs biologiques spécifiques des stades évolutifs de la toxoplasmose. Validation d'un modèle expérimental murin immunocompétent et immunodéprimé. Thèse pour le Doctorat en Biologie humaine Université Louis Pasteur, Strasbourg 1992.

Carrof B., Levacher-Clergeot M., Chau F., Pocidalo J.J. \& Derouin F. Toxoplasma gondii: Kinetics of lymphocyte subsets in blood and spleen of perorally infected mice. Experimental Parasitology, 1994, 78, 410-417.

Conley F.K., Jenkins K.A. \& Remington J.S. Toxoplasma gondii infection in the central nervous system : use of the peroxydase-antiperoxydase method to demonstrate toxoplasma in formalin-fixed embedded tissue section. Human Patbology, 1981, 12, 690-698.

Cristina N., Darde M.L., Boudin C., Tavernier G., PestreAlexandre M. \& Ambroise-Thomas P. A DNA fingerprint method for individual characterization of Toxoplasma gondii strains : combination with isoenzymatic characters for determination of linkage groups. Parasitology Research, 1995, 81, 32-37.

Craig W. Relevance of animal models for clinical treatment. European Journal of Clinical Microbiology and Infectious Diseases, 1993, 12 sp 1, 55-57.

Culberston W.W., Tabbara K. F. \& O'Connor G.R. Experimental ocular toxoplasmosis in primates. Archives of Ophthalmology, 1982, 100, 321-323.

DARCY F. and ZenNer L. Experimental models of toxoplasmosis. Research in Immunology, 1993, 144, 16-23.

Darde M.L., Bouteille B. \& Pestre-Alexandre M. Isoenzyme analysis of 35 Toxoplasma gondii isolates and the biological and epidemiological implications. Journal of Parasitology, 1992, 78, 786-794.

Davidson M.G., Lappin M.R., English R.V. \& Tompkins M.B. A feline model of ocular toxoplasmosis. Investigative Ophthalmology and Visual Science, 1993, 34, 3653-3660.

Davidson M.G., Rottiman J.B., English R.V., LApin M.R. \& Tompkins M.B. : Feline immunodefiency virus predisposes cats to acute generalized toxoplasmosis. American Journal of Pathology, 1993, 143, 1486-1497.

Derouin F., Almandany R., Chau F., Rouveix B. \& Pocidalo J.J. Synergistic activity of azithromycin and pyrimethamine or sulfadiazine in acute experimental toxoplasmosis. Antimicrobial Agents and Chemotherapy, 1992, 36, 997-1001.

Derouin F. \& GARIN Y.J.F. Toxoplasma gondii: blood and tissue kinetics during acute and chronic infections in mice. Experimental Parasitology, 1991, 73, 460-468.

Derouin F., Chau F., Caroff B., Romand S. \& Pocidalo J.J. : Bases expérimentales du traitement de la toxoplasmose. Médecine et Maladies Infectieuses, 1993, 23 S, 162-169.

Dubey J.P. \& Frenkel J.K. Feline toxoplasmosis from acutely infected mice and the development of Toxoplasma gondii. Journal of Protozoology, 1976, 23, 537-546.

DubeY J.P. \& SHEN S.K. Rat model of congenital toxoplasmosis. Infection and Immunity, 1991, 59, 3301-3302.

Dubey J.P., Schlafer D.H., Urban Jr. J.F. \& Lindsay D.S.
Lesions in fetal pigs with transplacentally-induced toxoplasmosis. Veterinary Pathology, 1990, 27, 411-418.

Dumas J.L., Chang H.R., Mermillod B., Piguet P.F., Comte R. \& PÉCHÈRE J.C. : Evaluation of the efficacy of prolonged administration of azithromycin in a murine model of chronic toxoplasmosis. Journal of Antimicrobial Chemotherapy, 1994, 34, 111-118.

Dutton G.N., Hay J. \& Kerrigan P. Photography of the retina in mice congenitally infected with Toxoplasma gondii. Annals of Tropical Medicine and Parasitology, 1984, 78, 435-437.

Dutton G.N., Hay J. \& Ralston J. The immunocytochemical demonstration of Toxoplasma within the eyes of congenitally infected mice. Annals of Tropical Medicine and Parasitology, 1984, 78, 431-433.

Ferguson D.J.P., Huskinson-Mark J., Araujo F.G. \& Remington J.S. A morphological study of chronic cerebral toxoplasmosis in mice : Comparison of four different strains of Toxoplasma gondii. Parasitology Research, 1994, 80, 493-501.

Ferguson D.J.P., Huskinson-Mark J., Araujo F.G. \& Remington J.S. An ultrastructural study of the effect of treatment with atovaquone in brains of mice chronically infected with the ME49 strain of Toxoplasma gondii. International Journal of Experimental Pathology, 1994, 75, 111-116.

Filice G.A. \& Pomeroy C. Effect of clindamycin on pneumonia from reactivation of Toxoplasma gondii infections in mice. Antimicrobial Agents and Chemotherapy, 1991, 35, 780-782.

Foulet A., Zenner L., Darcy F., Cesbron-Delauw M.F., Capron A. \& Gosselin B. Pathology of Toxoplasma gondii infection in the nude rat. Pathology and Research Practice, 1994, 190, 775-781.

Frenkel J.K., Nelson B.M. \& Arias-Stella J. Immunosuppression and toxoplasmic encephalitis. Human Patbology, 1975, 6, 97-111.

Gazzinelli R., Xu Y., Hieny S., Cheever A. \& Sher A. Simultaneous depletion of CD4+ and CD8+ T lymphocytes is required to reactivate chronic infection with Toxoplasma gondii. Journal of Immununology, 1992, 149, 175-180.

Gazzinelli R.T., Brézin A., Li Q., Nussenblatt R.B. \& Chan C.C. Toxoplasma gondii : acquired ocular toxoplasmosis in the murine model, protective role of TNF- $\alpha$ and INF- $\gamma$. Experimental Parasitology, 1994, 78, 217-229.

Gazzinelli R.T., Makino M., Chattopadhyay S.K., Snapper C.M., HÜGIN A.W. \& Morse H.C. Opportunistic infections and retrovirus-induced immunodeficiency : studies of acute and chronic infections with Toxoplasma gondii in mice infected with LP-BM5 murine leukemia viruses. Infection and Immunity, 1992, 60, 4394-4401.

Hay J., Lee W.R., Dutton G.N., Hutchison W.M. and Sim J.C. Congenital toxoplasmic retinochoroiditis in a mouse model. Annals of Tropical Medicine and Parasitology, 1984, 78, 109-116.

HitT J.A. \& Filice G.A. Detection of Toxoplasma gondii parasitemia by gene amplification, cell culture and 
mouse inoculation. Journal of Clinical Microbiology, 1992, 30, 3181-3184.

Hofflin J.M. \& Remington J.S. Clindamycin in a murine model of toxoplasmic encephalitis. Antimicrobial Agents and Chemotherapy, 1987, 31, 492-496.

Hofflin J.M. \& REMington J.S. In vivo synergism of roxithromycin (RU 965) and interferon against Toxoplasma gondii. Antimicrobial Agents and Chemotherapy, 1987, 31, 346-348.

Hofflin J.M., Conley F.K. \& Remington J.S. : Murine model of intracerebral toxoplasmosis. Journal of Infectious Diseases, 1987, 155, 550-557.

Holland G.N., O'Connor G.R., Diaz R.F., Minasi P. \& Waro W.M. Ocular toxoplasmosis in immunosuppressed nonhuman primates. Investigative Ophthalmology and Visual Science, 1988, 29, 835-842.

HOWE D.K. \& SiBley D. L. Toxoplasma gondii : analysis of different laboratory stocks of the RH strains reveals genetic heterogeneity. Experimental Parasitology, 1994, 78, 242-245.

Huldt G. Experimental toxoplasmosis : studies of the multiplication and spread of Toxoplasma in experimentally infected rabbits. Acta Patbologica et Microbiologica Scandinavica., 1966, 67, 401-423.

Huld G. Experimental toxoplasmosis transplacental transmission in guinea pigs. Acta Pathologica et Microbiologica Scandinavica, 1960, 49, 176-188.

Hutchison W.M., Hay J., LeE W.R. \& SiIm J.C. A study of cataract in murine congenital toxoplasmosis. Annals of Tropical Medicine and Parasitology, 1982, 76, 53-70.

Huskinson-Mark J., Araujo F.G. \& Remington J.S. Evaluation of the effect of drugs on the cyst form of Toxoplasma gondii. Journal of Infectious Diseases, 1991, 164, 170-177.

JOHNSON L.L. SCID mouse models of acute and relapsing chronic Toxoplasma gondii infections. Infection and Immunity, 1992, 60, 3719-3724.

Johnson A.M. Strain dependent, route of challenge-dependent susceptibility to toxoplasmosis. Zeitschrift für Parazitenkunde, 1984, 70, 303-309.

Joss A.W.L., Chatterton J.M.W., Evans R. \& Ho-Yen D.O. Toxoplasma polymerase chain reaction on experimental blood samples. Journal of Medical Microbiology, 1993, $38,38-43$.

Lacroix C., Levacher-Clergeot M., Chau F., Sumyuen M.H., Sinet M., Pocidalo J.J. \& Derouin F. Interactions between murine AIDS (MAIDS) and toxoplasmosis in co-infected mice. Clinical and Experimental immunology, 1994, 98, 190-195.

Lappin M.R., Gasper P.W., Rose B.J. \& Powell C.C. : Effect of primary phase feline immunodeficiency virus infection on cats with chronic toxoplasmosis. Veterinary Immunology and Immunopathology, 1992, 35, 121-131.

Lecomte V., Chumpitazi B., Pasquier B., Ambroise-Thomas P. \& SANTORO F. Brain tissue cyst in rats infected with RH strain of Toxoplasma gondii. Parasitology Research, 1992, 78, 267-269.

Lin D.S., BOWMAN D.D. \& JaCOBSON R.H. Immunological changes in cats with concurrent Toxoplasma gondii and
Feline Immunodeficiency Virus infections. Journal of Clinical Microbiology, 1992, 30, 17-24.

Maslo C., Perronne C., Della Bruna C., Pocidalo J.J. \& Derouin F. Synergistic activity of atovaquone against chronic Toxoplasma gondii and Mycobacterium avium intracellulare infection. 24th ICAAC, 1995, San Francisco (accepté)

McLeod R., Eisenhauer P., Mack D., Brown C., Filice G. \& SPITALNY G. Immune response associated with early survival after peroral infection with Toxoplasma gondii. Journal of Immunology, 1989, 142, 3247-3255.

McMenamin P.G., Dutton G.N., Hay J. \& Cameron S. The ultrastructural pathology of congenital murine toxoplasmic retinochoroiditis. Part I : the localization and morphology of Toxoplasma cysts in the retina. Experimental Eye Research, 1986, 43, 529-543.

Murray H.W., Teitelbaum R. \& Hariprashad J. Response to treatment for an intracellular infection in a $\mathrm{T}$ cell-deficient host : toxoplasmosis in nude mice. Journal of Infectious Diseases, 1993, 167, 1173-1177.

Newman P.E., Ghosheh R., Tabbara K.F., O'Connor G.R. \& STERN W. The role of hypersensitivity reactions to Toxoplasma antigens in experimental ocular toxoplasmosis in nonhuman primates. American Journal of Ophthalmology, 1982, 94, 159-164.

Nozik R.A. \& O'ConNor G.R. Experimental toxoplasmic retinochoroiditis. Archives of Ophthalmology, 1968, 79, 485489.

Olle P., Bessière M.H., Cassaing S., Estève T., Cazabonne P. \& Seguela J.P. Experimental murine toxoplasmic retinochoroiditis. The Journal of Eukaryotic Microbiology, 1994, $41,16 \mathrm{~S}$

Paugam A., Dupouy-Camet J., Sumyuen M.H., Romand S., LAmoril J. \& Derouin F. Detection of Toxoplasma gondii parasitaemia by polymerase chain reation in perorally infected mice. Parasite, 1995, 2, 181-184.

Piketty C., Derouin F., Rouveix B. \& Pocidalo J.J. In vivo assessment of antimicrobial agents against Toxoplasma gondii by quantification of parasites in the blood, lungs, and brain in infected mice. Antimicrobial Agents and Chemotherapy, 1990, 34, 1467-1472.

Pomeroy C., Filice G.A., Hitt J.A. \& Jordan M.C. Cytomegalovirus-induced reactivation of Toxoplasma gondii pneumonia in mice : lung lymphocyte phenotypes and suppressor function. Journal of Infectious Diseases, 1992, 166, 677-681.

Rollins D.F., Tabbara K.F., Ghosheh R. \& Nozik R.A. Minocycline in experimental ocular toxoplasmosis in the rabbit. American Journal of Ophthalmology, 1982, 93, 361-365.

Sasseville V.G., Pauley D.R., MacKey J.J. \& Simon M.A. Concurrent central nervous system toxoplasmosis and simian immunodeficiency virus-induced AIDS encephalomyelitis in a barbary macaque (Macaca sylvana). Veterinary Pathology, 1995, 32, 81-83.

SChlÜter D., LÖhler J., DeCKert M., HoF H. \& SChwendemanN G. Toxoplasma encephalitis of immunocompetent and nude mice : immunohistochemical characterisation of 
Toxoplasma antigen, infiltrates and major histocompatibility complex gene products. Journal of Neuroimmuno$\log y, 1991,31,185-198$.

Schoondermark-van de Ven E., Melchers W., Camps W., Eskes T., Meuwissen J. \& Galama J. Effectiveness of spiramycin for the treatment of congenital Toxoplasma gondii infection in rhesus monkeys. Antimicrobial Agents and Chemotherapy, 1994, 38, 1930-1936.

Schoondermark-van de Ven E., Galama E.J., Camps W., Vree T., Meuwissen J., \& Melchers W. : Pharmacokinetics of spiramycin in the rhesus monkeys : transplacental passage and distribution in tissue in the fetus. Antimicrobial Agents and Chemotherapy, 1994, 38, 1922-1929.

Schoondermark-van de Ven E., Galama J., Vree T., Camps W., BaArs I., Eskes T., Meuwissen J. \& Melchers W. : Study of treatment of congenital Toxoplasma gondii infection in rhesus monkeys with pyrimethamine and sulfadiazine. Antimicrobial Agents and Chemotherapy, 1995, 39, 137144.

Schoondermark-van de Ven E., Melchers W., Galama J., Camps W., Eskes T. \& Meuwissen J. : Congenital toxoplasmosis : an experimental study in rhesus monkeys for transmission and prenatal diagnosis. Experimental Parasitology, 1993, 77, 200-211.

Skorich D.N., Chiappino L. \& Nichols B.A. Invasion of the guinea pig conjunctiva by Toxoplasma gondii. Investigative Ophthalmology and Visual Science, 1988, 29, 1871-1880.

STAHL W. \& Turek G. Chronic murine toxoplasmosis : Clinicopathologic characterization of a progressive wasting syndrome. Annals of Tropical Medicine and Parasitology, 1988, 82, 35-48.

Stahl W., Matsubayashi H. \& AkaO S. Modification of subclinical toxoplamosis in mice by cortisone, 6-mercaptopurine and splenectomy. American Journal of Tropical Medicine and Hygiene, 1966, 27, 231-238.

Sumyuen M.H. Infection expérimentale par Toxoplasma gondii. Evolution chez l'hôte immunocompétent et immunodéprimé. Thèse de doctorat, Paris, 1995.

Sumyuen M.H., Garin Y.J.F. \& Derouin F. Early kinetics of Toxoplasma gondii infection in mice infected orally with cysts of an avirulent strain. Journal of Parasitology, 1995, $81,327-329$.

Suzuki Y., Conley F.K. \& Remington J.S. Interferon- $\gamma$ : the major mediator of resistance against Toxoplasma gondii. Science, 1988, 240, 516-518.

Suzuki Y., Conley F.K. \& Remington J.S. Treatment of toxoplasmic encephalitis in mice with recombinant gamma interferon. Infection and Immunity, 1990, 58, 3050-3055.

TABbara K.F., Nozik R.A. \& O'Connor G.R. Clindamycin effects on experimental ocular toxoplasmosis in the rabbit. Archives of Ophthalmology, 1974, 92, 244-247.

VAN DER WAAIJ D. Congenital transmission of avirulent Toxoplasma gondii after experimental infection in mice prior to gestation. Tropical and Geographical Medicine, 1960, 12, 251-257.

Wastling J.M., Nicoll S. \& Buxton D. Comparison of two gene amplification methods for the detection of
Toxoplasma gondii in experimentally infected sheep. Journal of Medical Microbiology, 1993, 38, 360-365.

Watanabe H., Suzuki Y., Makino M. \& Fujiwara M. Toxoplasma gondii: Induction of toxoplasmic encephalitis in mice with chronic infection by inoculation of a murine leukemia virus inducing immunodeficiency. Experimental Parasitology, 1993, 76, 39-45.

Weiss L.M., Udem S.A., Salgo M., Tanowitz H.B. \& Wittner M. Sensitive and specific detection of Toxoplasma DNA in an experimental murine model : use of Toxoplasma gondii-specific cDNA and the polymerase chain reaction. Journal of Infectious Diseases, 1991, 163, 180-186.

ZAK O. \& O'REILLY T. Animal models as predictors of the safety and efficacy of antibiotics. European Journal of Microbiology and Infectious Diseases, 1990, 9, 472-478.

Zenner L., Darcy F., Cesbron-Delaw M.F. \& Capron A. Rat model of congenital toxoplasmosis : rate of transmission of three Toxoplasma gondii strains to fetuses and protective effect of a chronic infection. Infection and Immunity, 1993, 61, 360-363. 